\title{
Detoxification of Implant Surfaces Affected by Peri-Implant Disease: An Overview of Non-surgical Methods
}

\author{
Pilar Valderrama ${ }^{1, *}$, Jonathan A Blansett ${ }^{1}$, Mayra G Gonzalez ${ }^{2}$, Myrna G Cantu ${ }^{3}$ and Thomas G Wil- \\ son $^{4}$ \\ ${ }^{I}$ Department of Periodontics, Texas A \& M University, Baylor College of Dentistry, Dallas, Texas 3302 Gaston Avenue, \\ Dallas Texas 75246 \\ ${ }^{2}$ Universidad de Panama, Facultad De, Odontologia Estafeta Universitaria Apartado 3366, Panama City, Panama, \\ 3366 \\ ${ }^{3}$ Universidad Autonoma de Nuevo Leon, Dr. Eduardo Aguirre Pequeno Street, Mitras Centro Monterrey, Mexico 64460 \\ ${ }^{4}$ Private Practice of Periodontics, 5465 Blair Rd, Ste 200, Dallas Texas 75231
}

\begin{abstract}
Objective: The aim of this review is to summarize the findings of studies that have evaluated non-surgical approaches for detoxification of implant body surfaces in vitro and in vivo, and to evaluate clinical trials on the use of these methodologies for treating peri-implant disease.

Materials and methods: A literature search was conducted using MEDLINE (Pubmed) from 1966 to 2013. In vitro and in vivo studies as well as clinical trials on non-surgical therapy were evaluated. The outcome variables were the ability of the therapeutic method to eliminate the biofilm and endotoxins from the implant surface, the changes in clinical parameters including probing depth, clinical attachment levels, bleeding on probing; radiographic bone fill and histological reosseointegration.

Results: From 134 articles found 35 were analyzed. The findings, advantages and disadvantages of using lasers as well as mechanical and chemical methods are discussed. Most of the in vivo and human studies used combination therapies which makes determining the efficacy of one specific method difficult. Most human studies are case series with short term longitudinal analysis without survival or failure reports.

Conclusion: Complete elimination of the biofilms is difficult to achieve using these approaches. All therapies induce changes of the chemical and physical properties of the implant surface. Re-osseointegration may be difficult to achieve if not impossible without surgical access to ensure thorough debridement of the defect and detoxification of the implant surface. Combination protocols for non-surgical treatment of peri-implantitis in humans have shown some positive clinical results but long-term evaluation to evaluate the validity and reliability of the techniques is needed.
\end{abstract}

Keywords: Detoxification, lasers, local drug delivery, non-surgical periodontal therapy, peri-implantitis.

\section{INTRODUCTION}

The vast majority of dental implants are successful over the long term. However, failure does occur. The two most common reasons for implant failure are mechanical (macro or micro motion) and infection. Peri-implant disease is initiated by bacteria and is differentiated into two subtypes, peri-implant mucositis and peri-implantitis $[1,2]$. By definition continued bone loss is seen in peri-implantitis but not in peri-implant mucositis. It is currently assumed that some cases of peri-implant mucositis will lead to peri-implantitis.

*Address correspondence to this author at the Department of Periodontics, Texas A \& M University, Baylor College of Dentistry, Dallas, Texas 3302 Gaston Avenue, Dallas Texas 75246; Tel: 214-828-8140;

Fax: 214-874-4563; E-mail: valderrama@bcd.tamhsc.edu
A recently published meta-analysis reported that periimplant, mucositis affects $63.4 \%$ of patients and $30.7 \%$ of implants while peri-implantitis affects $18.8 \%$ of patients and $9.6 \%$ of implants [2]. Peri-implantitis is frequently associated with implant failure. In order to reduce the number of implant failures the profession must develop appropriate methods for treating these disease states.

Peri-implant diseases have been associated with bacterial plaque [3]. Once these biofilms form on the implant surface removal of the bacteria and their byproducts such as lipopolysaccharides has proven problematic. In a previous paper by Valderrama and Wilson published in 2013, surgical methods for detoxification of implant surfaces were covered in detail [4]. The aim of this literature review is to summarize the available evidence about the non-surgical approaches to detoxify the contaminated implant surface. 


\section{MATERIALS AND METHODOLOGY}

A literature search was performed using MEDLINE (Pubmed) from January 1, 1966 to September 1, 2013. The search strategy is the same that was used for our recently published article about the surgical approach for detoxification of implants [4]. Briefly, the search included the following terms: non-surgical peri-implantitis treatment; implant surface decontamination and non-surgical treatment of mucositis. Articles in English were included and the search resulted in 134 articles. Titles and abstracts were screened and the full text of 69 publications reporting on the evaluation of mechanical, chemical and lasers used for the non-surgical treatment of contaminated implant surfaces were selected.

The following type of reports were included: in vitro and in vivo studies, case series, cohorts, systematic reviews and meta analysis. The bibliographies of systematic reviews and meta analysis were hand searched. Clinical studies reporting on the surgical treatment of peri-implantitis were excluded. The outcome variables were the ability of the therapeutic method to eliminate the biofilm and endotoxins from the implant surface, the changes in clinical parameters like probing depth (PD), clinical attachment levels (CAL), bleeding on probing (BOP), radiographic bone fill and histological reosseointegration as reported in in vivo studies.

\section{RESULTS}

Commonly used methods for non-surgical implant surface detoxification.

\section{MECHANICAL METHODS}

\section{Implantoplasty}

Implantoplasty is a technique to flatten/smooth the contaminated implant surface using rotary instruments [5]. This approach was proposed by Lang et al. [6] and reported by Suh et al. [7], and seeks to reduce the roughness of the implant surface which facilitates the colonization of biofilms [8-10] because it has been demonstrated that smoother surfaces retain less bacteria [11]. Rimondini et al. (2000) tested fifteen polishing procedures in vitro. They included carbide burs and diamond burs and demonstrated that all procedures resulted in smoother surfaces than at baseline under the scanning electron microscope. However, they reported that the best sequence was first using 30 microns and 15 microns mean-particle-size diamond burs followed by carbide 12 plus 16 bladed burs. They also reported that diamond burs produced granular debris and that carbide bladed burs produced needle or flake shaped debris [12]. Under proper cooling conditions, implantoplasty does not generate excess temperature increases that can damage soft tissue or bone surrounding the treated implant [13]. In vitro studies have shown that the use of diamond polishing devices can remove the coating of the implant surface entirely thus exposing the body of the fixture [14]. The exposure of the bulk of the implant could put the implant at risk for corrosion and therefore this technique should be limited to cases in which there are no other alternatives [15]. The remnants of the coating of the implant are expected to remain as metal debris in the surrounding tissues [14] inducing a foreign body reaction that could re- sult in more inflammation and bone loss around the implant as has been found in human histological analysis performed by our group (study in progress unpublished data). Another disadvantage of this technique is the increased post-operative soft tissue recession and exposure of the implant surface which affects esthetics [16].

\section{Air Powder Abrasives (AP)}

The use of an abrasive powder, like sodium bicarbonate, sodium hydrocarbonate [14], or the amino acid glycine [13], propelled by a stream of compressed air and water is called air powder abrasive [17]. This technique uses pressures of 65 to 100 pounds per square inch (psi) [18] and has been demonstrated with in vitro and in vivo studies to be effective in cleaning the previously contaminated implant surfaces [19]. Tastepe et al. (2012) concluded that this technique allows the removal of $84 \%$ to $98 \%$ of bacterial endotoxin from the implant surface and the removal of the bacteria biofilm up to $100 \%$ [17]. The use of AP for the treatment of peri-implant mucositis with sodium carbonate air-powder in combination with resin curettes resulted in a significant improvement in all clinical parameters. Tumor necrosis factor-alpha (TNFalpha) levels were significantly reduced achieving the same level as the healthy group at 3 months after therapies [20]. AP using amino acid glycine powder (AGP) has been compared to mechanical debridement using carbon curettes and antiseptic therapy with chlorhexidine digluconate. Both groups exhibited comparable PD reductions and CAL at 6 months, however, AGP showed significantly higher BOP reductions [21].

Some studies have shown that AP does not alter the physical structure of some implant surfaces, [22, 23] but others have shown that it could largely obliterate the milling marks and cause some surface pitting and irregular craterlike defects [24]. These changes are time dependent, in all specimens, a 5-second exposure did not induce deep changes in the surfaces but a 15-second exposure modified all the specimen surfaces [23]. Moreover, it has been shown that particles of the powder can stay attached to the implant surface after cleaning $[23,25,26]$.

It can be concluded that air powder abrasive can contribute to the detoxification of the implant surface and can produce a surface that is smoother than the original [5]. Negative adverse effects like subcutaneous emphysema and epithelial desquamation have been reported with the use of air abrasive around teeth [18] and around implants [27]. This potential complication may be prevented if the tip if the instrument is used at a $45^{\circ}$ angle to the implant [14].

\section{Ultrasonic Scalers With a Metal Tip}

When applied to rough surfaces, an ultrasonic scaler with a metal tip has been shown in vitro to produce a smoother surface with reduced irregularities and to remove bacteria more efficiently than ultrasonic scalers covered with a plastic tip [28]. Ultrasonic tips have been shown to decrease plaque scores from $73 \%$ to $53 \%(\mathrm{p}<0.01)$ and bleeding scores $(\mathrm{p}<0.01)$, however, no differences in probing depths were identified [29]. There is a concern about temperature increase when this instrument is used subgingivally during a non-surgical approach. It has been demonstrated that sonic 
and ultrasonic scalers did not show increase in temperatures of the implants and that there is a satisfactory functioning of the cooling system [30]. Metal tips have been shown to smooth the roughened surface which could facilitate the removal of bacteria using personal oral hygiene [28]. Machined surfaces may be altered, however, the scratches of the surfaces do not significantly affect the amount of biofilm that adheres. In fact one in vivo study showed that reduction of the surface roughness, below a certain threshold $\mathrm{R}(\mathrm{a})$ (0.2 microns), has no major impact on the supra- and subgingival microbial composition [31]. Ultrasonic scalers with metal tips may change the topographic characteristics of the implant surface or abutment, however, the changes may be inconspicuous or may enhance future plaque removal and facilitate maintenance.

\section{Metallic Curettes}

These instruments cause alteration of the smooth surface [32] including pronounced irregularities and high substance removal [22], however, the damage observed is less at the SEM level when curettes made of titanium are used [5]. An in vitro study using a surface profilometer showed that metal curettes made of stainless steel alloys reduce the roughness of rough surfaced implants and decrease the attachment of Streptococcus sanguinis which is an important early colonizer in the oral cavity [26]. Another study showed that 30 minutes after treatment with stainless steel alloy curettes, Aggregatibacter actinomycetemcomitans (serotype a) (former Actinomyces actinomycetemcomitans), Lactobacillus acidophilus, Streptococcus anginosus, and Veillonella parvula were found at lower counts $(\mathrm{p}<0.001)$. However, no microbiological differences between baseline and 6-month samples were found for any species [33]. Scaling alone with stainless steel alloy curettes has been shown to have no effect on mucosal hyperplasia, nor bleeding on probing scores. Some slight effect has been reported on plaque scores at 4 weeks but they tend to return to baseline values at 12 weeks; however, probing depths, clinical attachment levels, and probing bone levels have shown to remain unchanged after the use of this therapy alone [34].

One advantage of stainless steel alloy curettes is that after 20 seconds of use they can remove superficial material from the rough surface of on average $0.83 \mu \mathrm{m}$. This compared to $0.19 \mu \mathrm{m}$ removed by titanium curettes and ultrasonic tips covered with plastic inserts [22]. This could be advantageous when considering that Wilson demonstrated in 2009 that in $81 \%$ of cases of peri-implantitis there was excess of cement attached to the implant surface [35]. Removal of this material is important but in most cases complete removal is difficult to achieve. Moreover, some cements are chemically toxic to the peri-implant tissues [36]. Therefore, the need of removing strongly attached materials (including cement) from the surfaces can be more important than the risk of increasing the roughness of the surface $[22,35]$ since it has been shown that biofilm accumulation on rough implant surfaces is not significantly increased in subgingival areas $(0.8 \%+/-1.0 \%)$ compared to supragingival areas $(17.3 \%+/$ $23.1 \%$ ) [37]. Also, the use of metallic curettes for cement removal may be advantageous compared to the use of ultrasonic devices, because the use of ultrasonics may leave small particles of cement in the peri-implant tissues. Unpublished results by our group have shown that these small particles of cement are routinely surrounded by inflammatory cells and may contribute to peri-implant disease.

\section{Non-Metallic Curettes}

These instruments can be made of plastic, carbon, resinreinforced and resin-un-reinforced. An in vivo study in which peri-implant mucositis was induced in monkeys, showed that acrylic curettes and polishing with rubber cups and paste resulted in improvement of clinical parameters and minimal inflammation histologically [38]. However, with induced peri-implantitis in dogs where there was $40 \%$ bone loss present the non-surgical treatment with plastic curettes showed limited improvement and resulted in just $1 \%$ reosseointegration histologically [39]. In humans, the same approach also has proven to be beneficial for the treatment of peri-implant mucositis, plaque reduction and $\mathrm{BOP}$ reduction [40] but not for the treatment of peri-implantitis [41]. In a study in which carbon fiber curettes were used for subgingival debridement, plaque, BOP, PD, and bone levels remained unchanged 3 and 6 months after treatment [41]. The main advantage of non-metallic curettes is that they do not cause significant alteration of the surface when used to treat smooth surfaced implants [5]. However, when used several times the cumulative effect could result in a rougher surface [42]. A possible undesirable secondary effect is that when using non-metallic curettes to clean rough surfaces some of the curette material is deposited on the implant surface [5, $30,43]$. The effect of this debris is unknown, however, some plastic particles have been observed in human biopsies from patients with peri-implantitis (study in progress, unpublished data) An additional concern is that curettes made from these materials have difficulty in removing hard accretions, such as cement, from the implant surface.

In vitro studies have shown incomplete removal of biofilm with non-metallic curettes [14]. This could be associated with the size of these curettes which could prevent exact placement and application and may result in inefficient plaque removal [5]. However when combination of mechanical therapy with carbon fiber curettes and local antibiotics are used has shown to improve clinical parameters like $\mathrm{PD}$ and $\mathrm{BOP}$ [44].

\section{Rubber Cups}

Rubber cups have shown to generate significant smoothening of the titanium surface and significantly decrease roughness by removing surface debris and rounding off the sharp machined grooves present on the untreated abutment surface [32]. In another in vitro study it was shown that the rubber cup, the plastic curette, and AP left the implant surfaces unchanged [22]. Polishing the implant surfaces with pumice and a rubber cup combined with irrigation with chlorhexidine and systemic antibiotics results in reduction of anaerobic bacteria and bleeding scores in patients with periimplantitis [45]. 


\section{CHEMICAL METHODS}

\section{Citric Acid (CA)}

In vitro, $\mathrm{CA} \mathrm{pH} 1$ has been shown to significantly decrease the amount of Escherichia coli LPS after burnishing with a cotton pellet for 1 minute on titanium alloy grit blasted and on hydroxyapatite (HA) coated strips [46]. CA also has shown to reduce Porphyromonas gingivalis endotoxin when applied for one minute or 2 minutes in vitro. The one minute treatment lead to a reduction of the endotoxin by up to $85.8 \%$ for machined surface, $27 \%$ for titanium plasma sprayed and $86.8 \%$ for hydroxyapatite coated titanium implants. The reduction after 2 minutes of CA application was $90 \%, 36.4 \%$ and $92.1 \%$ respectively [47]. However, CA was unable to inactivate bacterial biofilms formed on smooth titanium discs intraorally in humans after submerging the discs in a 40\% CA solution for 1 minute [48]. This could be the result of the characteristics of biofilms that are protected by glycocalix or the fact that no mechanical disruption happened in the immersion study.

CA can alter the composition of the implant surface increasing the amount of ions of carbon, oxygen and nitrogen which could inhibit re-osseointegration [25]. CA has been shown to suppress fibroblast attachment in vitro [49], CA application therefore must be limited to the implant surface avoiding the spread of it to the bone and marrow spaces making clinical application of this material difficult. Also low $\mathrm{pH}$ acidic substances like CA have been related to implant surface corrosion, potentially reducing the chance for re-osseointegration [50].

\section{Chlorhexidine (CHX)}

Due to the bactericidal effect of CHX, it has been used frequently to try to decrease the bacteria in cases of periimplantitis. Subgingival irrigation with CHX $0.12 \%$ combined with mechanical debridement has shown to reduce bacteria and improve probing depths up to 3 months after therapy in humans [40]. In vitro, application of CHX (0.2\%) for 60 seconds has shown to be effective against Streptococcus sanguinis and Candida albicans [51]. Rubbing the implant surface for 1 minute with a cotton pellet soaked with $0.12 \%$ CHX reduces the amount of Porphyromonas gingivalis endotoxin on machined surfaces up to $92.9 \%$, on titanium plasma sprayed surfaces to $62.9 \%$ and on HA coated surface to $62.8 \%$ [47]. Topical application of $0.2 \%$ CHX gel after mechanical cleaning combined with a $1 \mathrm{mg}$ total dose of minocycline microspheres into the peri-implant defect has been shown to successfully reduce the microbiota in $48 \%$ of the cases, however, authors reported that $32 \%$ of the subjects did not respond to this therapy resulting in either implant loss or increased bacterial load to baseline levels [52]. $1 \% \mathrm{CHX}$ gel as an adjunctive subgingival antimicrobial treatment results in limited reduction of bleeding scores at 12 months [53].

The cell toxicity of CHX is influenced by concentration and exposure time. No osteoblast phenotypic alteration has been observed after exposure to $0.2 \%$ CHX for 1 minute and CHX $1 \%$ for 30 seconds [54]. The clinical application of
$\mathrm{CHX}$ as evidenced in this review requires longer periods of time than 30 seconds or 1 minute. CHX has been shown to induce apoptosis and cell necrosis due to disturbance of mitochondrial function, intracellular $\mathrm{Ca}^{2+}$ increase and oxidative stress [55]. CHX also has shown to inhibit cell proliferation and collagen synthesis [56]. These negative effects could explain the reduction in bacterial counts but no significant improvement in tissue healing.

\section{Hydrogen Peroxide (HP)}

Rubbing with a cotton pellet soaked with 3\% HP for 1 minute in vitro has been shown to significantly decrease the amount of Escherichia coli LPS (LPS count 108 $\mathrm{min} / \mathrm{mm}^{2}$ ) on titanium alloy grit blasted and HA coated strips compared to untreated controls (LPS count $197 \mathrm{~min} / \mathrm{mm}^{2}$ ). However, HP was the least effective when compared to citric acid, plastic sonic scaler tips and air powder abrasive [46]. In another in vitro study designed to evaluate the ability of HP to eliminate Candida albicans, Streptococcus sanguinis, or Staphylococcus epidermidis from titanium specimens, HP alone was effective against Candida albicans [51]. 3\% HP was capable of inactivating attached bacterial cells from human biofilms after immersion in HP for 1 minute [48]. 10\% HP has also shown to inactivate a human biofilm created in the lab and to eliminate $99.9 \%$ of the bacteria attached to the implant surface [57]. Swabbing the implant surface with $10 \%$ HP for 1 minute has also been shown in animals to decontaminate the implant surface and to allow re-osseointegration to previously contaminated surface in dogs [58].

\section{Lasers}

Many different types of lasers have been investigated including but not limited to diode, carbon dioxide $\left(\mathrm{CO}_{2}\right)$, neodymium doped yttrium aluminum garnet (Nd:YAG), erbium, yttrium aluminum, garnet (Er:YAG), and gallium aluminum arsenide (GaAlAs). In vitro studies have demonstrated the ability of lasers to lower total bacterial counts on contaminated titanium surfaces. In an in vitro study, Nd:YAG and GaAlAs lasers were demonstrated to sterilize implant surfaces of Porphyromonas gingivalis and Escherichia faecalis with no surface topography changes when viewed under SEM [59]. However, laser energy at the wrong setting can alter and/or melt the surface of dental implants, potentially interfering with re-osseointegration. Many in vitro studies have been completed analyzing the effect on the implant surface after irradiation at varying power settings, modes, and application times. In one in vitro study sand blasted and acid-etched, plasma sprayed, hydroxyapetite-coated, and smooth surface titanium discs were treated with Nd:YAG, Holmium:YAG (Ho-YAG), Er:YAG, $\mathrm{CO}_{2}$, and GaAlAs lasers at a variety of power settings and viewed under SEM and energy dispersive spectroscopy. The authors found that the pulsed Er-YAG, Ho-YAG and Nd-YAG lasers induced implant surface changes in an energy-dependent manner. Within the study parameters, the $\mathrm{CO}_{2}$ induced surface changes in the hydroxyapetite, sand blasted and acid etched, and plasma sprayed discs. GaAlAs irradiation did not damage any of the disc surfaces. Based on their findings, the authors recommended that Nd:YAG and Ho:YAG lasers can be damaging to the implant surfaces and should not be used. 
Also, the Er:YAG and $\mathrm{CO}_{2}$ lasers may be useful but the power output should be limited to avoid surface alterations and finally that the GaAlAs laser was safe with respect to surface alterations [60]. In another in vitro study, the surface alterations on machined and sand-blasted, large grit, acidetched (SLA) titanium discs after laser irradiation with a $\mathrm{CO}_{2}$, diode, and Er:YAG laser were analyzed by SEM and light confocal microscopy. No surface alterations on either type of disc were observed after irradiation with a $\mathrm{CO}_{2}$ or diode laser for any of the chosen study parameters. In contrast, the Er:YAG laser caused surface alterations in both SLA and polished discs. The SLA surfaces showed changes after 10 seconds of treatment at $300 \mathrm{~mJ} / 10 \mathrm{~Hz}$. The polished surfaces showed surface alterations after 10 seconds of irradiation at $500 \mathrm{~mJ} / 10 \mathrm{~Hz}$. The authors concluded that if an Er:YAG laser was to be used, power settings should be limited to below $300-500 \mathrm{~mJ} / 10 \mathrm{~Hz}$ [61]. Another in vitro study analyzed the effects of different power and cooling methods when an Er:YAG laser was used on anodically etched, medium rough (RP MKIII TiUnite $3.75 \mathrm{~mm} \times 13 \mathrm{~mm}$, Nobel Biocare) titanium implant surfaces. The authors found that a power setting of $100 \mathrm{~mJ}$ with a wavelength of $10 \mathrm{~Hz}$ was optimal and was able to evenly strip a layer of titanium oxide from the implant surface without causing thermal damage to the newly exposed layer of titanium oxide. At this power setting with water spray, the maximum the implant surface was heated was 3 Celsius degrees, whereas without water spray the temperature increased by 30-33 Celsius degrees. Also, when the authors performed the same protocol with a $\mathrm{CO}_{2}$ laser with air cooling, the implant surfaces were heated by 50 Celsius degrees [62]. An in vivo study by Schwarz et al. (2005) in humans analyzed treatment of peri-implant defects in twenty patients with 40 contaminated implants. 20 implants were decontaminated non-surgically with an Er:YAG laser (wavelength $10 \mathrm{~Hz}$, power $100 \mathrm{~mJ}$ ), and 20 implants were treated with mechanical debridement with plastic curets and a $0.2 \%$ CHX rinse subgingivally. Both groups had significant beneficial changes in BOP, PD, and CAL at 3 and 6 month recall exams, however these gains were not maintained at the 12-month recall exam, except for sustained significantly lower reductions in BOP in the laser group compared to the control group [63]. In another in vivo study in humans, the use of an Er:YAG laser in conjunction to S/RP was compared to Er:YAG treatment alone in a split mouth protocol in 20 patients. Clinical and microbiologic parameters were assessed at baseline and at 3, 6, and 12 months post-treatment. The Er:YAG laser was set at $160 \mathrm{~mJ}$ at a repetition rate of $10 \mathrm{~Hz}$. The authors reported statistically significant reductions in plaque scores, gingival index, and BOP at each recall appointment with each treatment modality. Mean probing depths decreased from $5.2 \mathrm{~mm}$ to $3.2 \mathrm{~mm}$ after 12 months in the laser treatment group alone and from $5.0 \mathrm{~mm}$ to $3.3 \mathrm{~mm}$ in the laser $+\mathrm{S} / \mathrm{RP}$ group. Gains in CAL for both the laser treated and laser treated $+\mathrm{S} / \mathrm{RP}$ sites were $1.6 \mathrm{~mm}$. Both treatments resulted in a significant increase of cocci and non-motile rods and a decrease in motile rods and spirochetes. No significant differences between sites were observed at any time point [64]. In another human study, Renvert et al. assessed treatment of peri-implantitis lesions in 21 patients using an Er:YAG laser or AP with a subgingival application tip in a non-surgical approach. At the end of the 6-month study period, no significant differences in clinical outcomes was observed between either treatment modality with respect to changes in PD, BOP, and CAL [65]. While some laser applications have shown promising results, at present specific conclusions cannot be drawn concerning type of laser, power settings and appropriate clinical scenarios for their use.

\section{Photodynamic Therapy (PDT)}

PDT involves coating a substance (implant surface, bacterial cells, etc) with a particular photosensitizing agent with a known wavelength of activation. Once activated by light of the excitation wavelength in the presence of oxygen, the photosensitizer transitions from a low-energy ground state to a high-energy singlet state, causing a production in free radicals that are toxic to target cells [66]. It has also been demonstrated in vitro that lethal photosensitization of bacteria can be achieved without damage to the implant surface [67]. It has also been shown in vitro that photosensitization and light activation is more effective in killing bacteria from titanium surfaces than laser ablation alone [67]. In an in vivo study by Schär et al., forty patients with one affected implant were treated by photodynamic therapy with a diode laser (wavelength $660 \mathrm{~nm}$, power $100 \mathrm{~mW}$ ) was compared to minocycline microsphere delivery ( $\mathrm{n}=20$ each group) after initial debridement with titanium curettes and AP with glycine powder. Phenothyazine chloride was applied as a photoactivator and activated with a 10 second laser application and this was repeated after 1 week. The minocycline treated implants had $1 \mathrm{mg}$ applied subgingivally. At sites with residual $\mathrm{BOP}$, the interventions were repeated at the 3 and 6-month recall exam. At 3 months, both interventions resulted in reduction of BOP (2.21 sites in PDT and 1.77 sites in the minocycline group). At 6 months, $15 \%$ of control implants had total resolution of inflammation where $30 \%$ of test implants had no BOP. All implants had statistically significant gains in CAL, reduction in PD and plaque at the 3 and 6-month recall exams, with no statistical difference between the two treatment modalities. The authors concluded that PDT is as effective as local delivery of minocycline in treating periimplantitis after initial therapy + glycine AP had been completed and these gains were maintained up to 6 months [68]. In conclusion, the use of photosensitizing dyes in conjunction with laser excitation may be beneficial in lowering bacterial counts from affected implants, however the short term evaluation periods in these studies do not allow to evaluate the arrest of the peri-implant lesion's progression long-term.

\section{DISCUSSION}

The goal of treating peri-implant diseases (peri-implant mucositis and peri-implantitis) is to stop the inflammatory process and if possible reverse the bone loss seen as a result of disease. If one starts with the assumption that these disease states are initiated and exacerbated by bacteria then removal of these microbiota and their byproducts becomes essential. This review has looked at currently used approaches to achieve this goal with non-surgical methods. We found that to date no one method or combination of methods applied non-surgically has routinely resulted in the elimination of these disease states. Our conclusion agree with the results of a network meta-analysis [69] and systematic re- 
views $[70,71]$. Most of the human studies published are case series with short term follow-up periods making it difficult to determine the stability of the resolution of the inflammation and decrease of pathogenic bacteria over time. Most of the human studies included in this review base their conclusion on clinical measurements like probing depths or clinical attachment levels, but do not report success or survival rates. Therefore it is difficult to determine what approach will improve implant survival. This is a common inconvenient found when analyzing the data about therapies for periimplant diseases [72, 73].

Care should be exercised in the use of acidic chemicals to detoxify implant surfaces, due to surface alterations in the titanium oxide layer that appears to be necessary for reattachment. The same can be said for certain approaches using lasers. However, within this last group some laser types at specific settings have showed early promise in detoxification. Combination of physical and chemical and laser therapy may provide more predictable results. In general, it should be noted that the profession is early in its understanding of these diseases and their treatment.

From this review it can be argued that further investigation of optimal ways to treat implants affected by periimplantitis and peri-implant mucositis as well as the prevention of these problems is warranted.

\section{CONCLUSION}

Complete elimination of the biofilms needed for reattaching bone to previously contaminated implant surfaces is difficult to achieve. All therapies induce changes of the implant surface chemical and physical properties. Combination protocols for non-surgical treatment of peri-implantitis in humans have shown some positive results but long-term evaluation to establish the validity and reliability of the techniques has yet to be concluded.

\section{CONFLICT OF INTEREST}

The authors confirm that this article content has no conflict of interest.

\section{ACKNOWLEDGEMENTS}

Dr. Thomas G. Wilson Jr. declares that works on some research projects for a company called: El.En. at Florence, Italy.

\section{REFERENCES}

[1] Froum SJ, Rosen PS. A proposed classification for peri-implantitis. Int J Periodontics Restorative Dent 2012; 32(5): 533-40.

[2] Atieh MA, Alsabeeha NH, Faggion CM Jr, Duncan WJ. The frequency of peri-implant diseases: a systematic review and metaanalysis. J Periodontol 2012 (Epub).

[3] Pontoriero R, Tonelli MP, Carnevale G, Mombelli A, Nyman SR, Lang NP. Experimentally induced peri-implant mucositis: a clinical study in humans. Clin Oral Implants Res 1994; 5(4): 254-9.

[4] Valderrama P, Wilson TG Jr. Detoxification of implant surfaces affected by peri-implant disease: an overview of surgical methods. Int J Dent 2013; 2013:740680.

[5] Louropoulou A, Slot DE, Van der Weijden FA. Titanium surface alterations following the use of different mechanical instruments: a systematic review. Clin Oral Implants Res 2012; 23(6): 643-58.
[6] Lang NP, Wilson TG, Corbet EF. Biological complications with dental implants: their prevention, diagnosis and treatment. Clin Oral Implants Res 2000; 11(1): 146-55.

[7] Suh JJ, Simon Z, Jeon YS, Choi BG, Kim CK. The use of implantoplasty and guided bone regeneration in the treatment of periimplantitis: two case reports. Implant Dent 2003; 12(4): 277-82.

[8] Quireynen M, VanDerMei HC, Steenberghe VD. An in vivo study of the influence of surface roughness of implants on the microbiology of the supra and subgingival plaque. J Dent Res 1993; 72: 1304-9.

[9] Subramani K, Jung R, Molenberg A, Hammerle C. Biofilm on dental implants: a review of the literature. Int J Oral Maxillofac Implants 2009; 24: 616-26.

[10] Romeo E, Ghisolfi M, Carmagnola D. Peri-implant diseases: a systematic review of the literature. Minerva Stomatol 2004; 53(5): 215-30.

[11] Rimondini L, Fare S, Brabilla E. The effect of surface roughness on early in vivo plaque colonization on titanium. J Periodontol 1997; 68(6): 556-62.

[12] Rimondini L, Cicognani Simoncini F, Carrassi A. Micromorphometric assessment of titanium plasma-sprayed coating removal using burs for the treatment of peri-implant disease. Clin Oral Implants Res 2000; 11(2): 129-38.

[13] Schwarz F, Ferrari D, Popovski K, Hartig B, Becker J. Influence of different air-abrasive powders on cell viability at biologically contaminated titanium dental implants surfaces. J Biomed Mater Res B Appl Biomater 2009; 88(1): 83-91.

[14] Augthun M, Tinschert J, Huber A. In vitro studies on the effect of cleaning methods on different implant surfaces. J Periodontol 1998; 69(8): 857-64.

[15] Rodrigues DC, Urban RM, Jacobs JJ, Gilbert JL. In vivo severe corrosion and hydrogen embrittlement of retrieved modular body titanium alloy hip-implants. J Biomed Mater Res B Appl Biomater 2009; 88: 206-19.

[16] Romeo E, Ghisolfi M, Murgolo N, Chiapasco M, Lops D, Vogel G. Therapy of peri-implantitis with resective surgery. A 3-year clinical trial on rough screw-shaped oral implants. Part I: clinical outcome. Clin Oral Implants Res 2005; 16: 9-18.

[17] Tastepe CS, van Waas R, Liu Y, Wismeijer D. Air powder abrasive treatment as an implant surface cleaning method: a literature review. Int J Oral Maxillofac Implants 2012; 27(6): 1461-73.

[18] Finlayson RS, Stevens FD. Subcutaneous facial emphysema secondary to use of the Cavi-Jet. J Periodontol 1988; 59(5): 315-7.

[19] Meyle J. Mechanical, chemical and laser treatments of the implant surface in the presence of marginal bone loss around implants. Eur J Oral Implantol 2012; 5 Suppl: S71-81.

[20] Duarte PM, de Mendonça AC, Máximo MB, Santos VR, Bastos MF, Nociti FH. Effect of anti-infective mechanical therapy on clinical parameters and cytokine levels in human peri-implant diseases. J Periodontol 2009; 80: 234-43.

[21] Schwarz F, Mihatovic I, Becker J, et al. Non-surgical treatment of peri-implantitis using an air-abrasive device or mechanical debridement and local application of chlorhexidine: a prospective, randomized, controlled clinical study. J Clin Periodontol 2011; 38(9): 872-8

[22] Mengel R, Buns C-E, Mengel C, Flores-de-Jacoby L. An in vitro study of the treatment of implant surfaces with different instruments. Int J Oral Maxillofac Implants 1998; 13(1): 91-6.

[23] Chairay JP, Boulekbache H, Jean A, Soyer A, Bouchard P. Scanning electron microscopic evaluation of the effects of an airabrasive system on dental implants: a comparative in vitro study between machined and plasma-sprayed titanium surfaces. J Periodontol 1997; 68(12): 1215-22.

[24] McCollum J, O'Neal RB, Brennan WA, Van Dyke TE, Horner JA. The effect of titanium implant abutment surface irregularities on plaque accumulation in vivo. J Periodontol 1992; 63(10): 802-5.

[25] Mouhyi J, Sennerby L, Pireaux JJ, Dourov N, Nammour S, Van Reck J. An XPS and SEM evaluation of six chemical and physical techniques for cleaning of contaminated titanium implants. Clin Oral Implants Res 1998; 9(3): 185-94.

[26] Duarte PM, Reis AF, Moreira de Freitas P, Ota-Tsuzuki C. Bacterial adhesion on smooth and rough titanium surfaces after treatment with different instruments. J Periodontol 2009; 80(11): 1824-32.

[27] Bergendal T, Forsgren L, Kvint S, Löwstedt E. The effect of an airbrasive instrument on soft and hard tissues around osseointegrated implants: a case report. Swed Dent J 1990; 14(5): 219-23. 
[28] Park JB, Jang YJ, Koh M, Choi BK, Kim KK, Ko Y. In vitro analysis of the efficacy of ultrasonic scalers and a toothbrush for removing bacteria from RBM titanium discs. J Periodontol 2012; 84(8): 1191-8.

[29] Renvert S, Samuelsson E, Lindahl C, et al. Mechanical nonsurgical treatment of peri-implantitis: a double-blind randomized longitudinal clinical study I: clinical results. J Clin Periodontol 2009; 36(7): 604-9.

[30] Ruhling A, Kocher T, Kreusch J, Plagmann HC. Treatment of subgingival implant surfaces with teflon-coated sonic and ultrasonic scaler tips and various implant curettes: an in vitro study. Clin Oral Implants Res 1994; 5(1): 19-29.

[31] Bollen CM, Papaioanno W, Van Eldere J, Schepers E, Quirynen M, van Steenberghe D. The influence of abutment surface roughness on plaque accumulation and peri-implant mucositis. Clin Oral Implants Res 1996; 7(3): 201-11.

[32] Homiak AW, Cook PA, DeBoer J. Effect of hygiene instrumentation on titanium abutments: a scanning electron microscopy study. J Prosthet Dent 1992; 67(3): 364-9.

[33] Persson GR, Samuelsson E, Lindahl C, Renvert S. Mechanical nonsurgical treatment of peri-implantitis: a single-blinded randomized longitudinal clinical study. II. microbiological results. J Clin Periodontol 2010; 37(6): 563-73.

[34] Schenk G, Flemmig TF, Betz T, Reuther J, Klaiber B. Controlled local delivery of tetracycline $\mathrm{HCl}$ in the treatment of peri-implant mucosal hyperplasia and mucositis: a controlled case series. Clin Oral Implants Res 1997; 8(5): 427-33.

[35] Wilson TG Jr. The positive relationship between excess cement and peri-implant disease: a prospective clinical endoscopic study. J Periodontol 2009; 80(9):1388-92.

[36] Wadhwani CP, Schwedhelm ER. The role of cements in dental lant success, Part I. Dent Today 2013; 32(4): 74-8; quiz 78-9.

[37] Elter C, Heuer W, Demling A, et al. Supra- and subgingival biofilm formation on implant abutments with different surface characteristics. Int J Oral Maxillofac Implants 2008; 23(2): 327-34.

[38] Trejo PM, Bonaventura G, Weng D, Caffesse RG, Bragger U, Lang NP. Effect of mechanical and antiseptic therapy on peri-implant mucositis: an experimental study in monkeys. Clin Oral Implants Res 2006; 17(3): 294-304.

[39] Schwarz F, Jepsen S, Herten M, Sager M, Rothamel D, Becker J. Influence of different treatment approaches on non-submerged and submerged healing of ligature induced peri-implantitis lesions: an experimental study in dogs. J Clin Periodontol 2006; 33(8): 584-95.

[40] Porras R, Anderson GB, Caffesse R, Narendran S, Trejo PM. Clinical response to 2 different therapeutic regimens to treat periimplant mucositis. J Periodontol 2002; 73(10): 1118-25.

[41] Karring ES, Stavropoulos A, Ellegaard B, Karring T. Treatment of peri-implantitis by the vector system. Clin Oral Implants Res 2005; 16(3): 288-93.

[42] Hallmon WW, Waldrop TC, Meffert RM, Wade BW. A comparative study of the effects of metallic, nonmetallic, and sonic instrumentation on titanium abutment surfaces. Int J Oral Maxillofac Implants 1996; 11(1): 96-100.

[43] Ramaglia L, di Lauro AE, Morgese F, Squillace A. Profilometric and standard error of the mean analysis of rough implant surfaces treated with different instrumentations. Implant Dent 2006; 15(1): 77-82.

[44] Salvi GE, Persson GR, Heitz-Mayfield LJ, Frei M, Lang NP. Adjunctive local antibiotic therapy in the treatment of peri-implantitis II: clinical and radiographic outcomes. Clin Oral Implants Res 2007; 18(3): 281-5.

[45] Mombelli A, Lang NP, Antimicrobial treatment of peri-implant infections. Clin Oral Implants Res 1992; 3(4): 162-8.

[46] Zablotsky MH, Diedrich DL, Meffert RM. Detoxification of endotoxin-contaminated titanium and hydroxyapatite-coated surfaces utilizing various chemotherapeutic and mechanical modalities. Implant Dent 1992; 1(2): 154-8.

[47] Dennison DK, Huerzeler MB, Quinones C, Caffese RG. Contaminated implant surfaces: an in vitro comparison of implant surface coating and treatment modalities for decontamination. J Periodontol 1994; 65(10): 942-8.

[48] Gosau M, Hahnel S, Schwarz F, Gerlach T, Reichert TE, Bürgers R. Effect of six different peri-implantitis disinfection methods on in vivo human oral biofilm. Clin Oral Implants Res 2010; 21(8): 86672 .
[49] Lan WC, Lan WH, Chan CP, Hsieh CC, Chang MC, Jeng JH. The effects of extracellular citric acid acidosis on the viability, cellular adhesion capacity and protein synthesis of cultured human gingival fibroblasts. Aust Dent J 1999; 44(2): 123-30.

[50] Frank MJ, Walter MS, Lyngstadaas SP, Wintermantel E, Haugen HJ. Hydrogen content in titanium and a titanium-zirconium alloy after acid etching. Mater Sci Eng C Mater Biol Appl 2013; 33(3): $1282-8$.

[51] Bürgers R, Witecy C, Hahnel S, Gosau M. The effect of various topical peri-implantitis antiseptics on Staphylococcus epidermidis, Candida albicans, and Streptococcus sanguinis. Arch Oral Biol 2012; 57(7): 940-7.

[52] Persson GR1, Salvi GE, Heitz-Mayfield LJ, Lang NP. Antimicrobial therapy using a local drug delivery system (Arestin) in the treatment of peri-implantitis. I: microbiological outcomes. Clin Oral Implants Res 2006; 17(4): 386-93.

[53] Renvert S, Lessem J, Dahlén G, Lindahl C, Svensson M. Topical minocycline microspheres versus topical chlorhexidine gel as an adjunct to mechanical debridement of incipient peri-implant infections: a randomized clinical trial. J Clin Periodontol 2006; 33(5): 362-9.

[54] Verdugo F, Sáez-Rosón A, Uribarri A, et al., Bone microbial decontamination agents in osseous grafting: an in vitro study with fresh human explants. J Periodontol 2011; 82(6): 863-71.

[55] Giannelli M, Chellini F, Margheri M, Tonelli P, Tani A.Effect of chlorhexidine digluconate on different cell types: a molecular and ultrastructural investigation. Toxicol In Vitro 2008; 22(2): 308-17.

[56] Lee TH, Hu CC, Lee SS, Chou MY, Chang YC. Cytotoxicity of chlorhexidine on human osteoblastic cells is related to intracellular glutathione levels. Int Endod J 2010; 43(5): 430-5.

[57] Ntrouka VI, Slot DE, Louropoulou A, Van der Weijden F. The effect of chemotherapeutic agents on contaminated titanium surfaces: a systematic review. Clin Oral Implants Res 2011; 22(7): 681-90.

[58] Alhag M, Renvert S, Polyzois I, Claffey N. Re-osseointegration on rough implant surfaces previously coated with bacterial biofilm: an experimental study in the dog. Clin Oral Implants Res 2008; 19(2): $182-7$.

[59] Gonçalves F, Zanetti AL, Zanetti RV, et al. Effectiveness of 980-mm diode and 1064-nm extra-long-pulse neodymium-doped yttrium aluminum garnet lasers in implant disinfection. Photomed Laser Surg 2010; 28(2): 273-80.

[60] Kreisler M, Gotz H, Duschner H. Effect of Nd:YAG, Ho:YAG, Er:YAG, $\mathrm{CO}_{2}$, and GaAIAs laser irradiation on surface properties of endosseous dental implants. Int J Oral Maxillofac Implants 2002; 17(2): 202-11.

[61] Stubinger S, Etter C, Miskiewicz M, et al. Surface alterations of polished and sandblasted and acid-etched titanium implants after Er:YAG, carbon dioxide, and diode laser irradiation. Int $\mathrm{J}$ Oral Maxillofac Implants 2010; 25(1): 104-11.

[62] Yamamoto A, Tanabe T. Treatment of peri-implantitis around TiUnite-surface implants using Er:YAG laser microexplosions. Int J Periodontics Restorative Dent 2013; 33(1): 21-30.

[63] Sculean A, Schwarz F, Becker J. Anti-infective therapy with an Er:YAG laser: influence on peri-implant healing. Expert Rev Med Devices 2005; 2(3): 267-76.

[64] Schwarz F, Rothamel D, Sculean A, Georg T, Scherbaum W, Becker J. Effects of an Er:YAG laser and the vector ultrasonic system on the biocompatibility of titanium implants in cultures of human osteoblast-like cells. Clin Oral Implants Res 2003; 14(6): 78492.

[65] Persson GR, Roos-Jansåker AM, Lindahl C, Renvert S. Microbiologic results after non-surgical erbium-doped:yttrium, aluminum, and garnet laser or air-abrasive treatment of peri-implantitis: a randomized clinical trial. J Periodontol 2011; 82(9): 1267-78.

[66] Takasaki AA, Aoki A, Mizutani K, et al. Application of antimicrobial photodynamic therapy in periodontal and peri-implant diseases. Periodontol 2000, 2009; 51: 109-40.

[67] Haas R, Dörtbudak O, Mensdorff-Pouilly N, Mailath G. Elimination of bacteria on different implant surfaces through photosensitization and soft laser. An in vitro study. Clin Oral Implants Res 1997; 8(4): 249-54.

[68] Schär D, Ramseier CA, Eick S, Arweiler NB, Sculean A, Salvi GE. Anti-infective therapy of peri-implantitis with adjunctive local drug delivery or photodynamic therapy: six-month outcomes of a pro- 
spective randomized clinical trial. Clin Oral Implants Res 2013; 24(1): 104-10.

[69] Faggion CM Jr, Chambrone L, Listl S, Tu YK. Network metaanalysis for evaluating interventions in implant dentistry: the case of peri-implantitis treatment. Clin Implant Dent Relat Res 2013; 15(4): 576-88

[70] Schwarz F, Iglhaut G, Becker J. Quality assessment of reporting of animal studies on pathogenesis and treatment of peri-implant mucositis and peri-implantitis: a systematic review using the ARRIVE guidelines. J Clin Periodontol 2012; 39(12): 63-72.
[71] Graziani F, Figuero E, Herrera D. Systematic review of quality of reporting, outcome measurements and methods to study efficacy of preventive and therapeutic approaches to peri-implant diseases. J Clin Periodontol 2012; 39(12): 224-44.

[72] Faggion CM Jr, Schmitter M. Using the best available evidence to support clinical decisions in implant dentistry. Int J Oral Maxillofac Implants 2010; 25(5): 960-9.

[73] Faggion CM Jr, Listl S, Tu YK. Assessment of endpoints in studies on peri-implantitis treatment--a systematic review. J Dent 2010; 38(6): $443-5$

Received: January 10, 2014

(C) Valderrama et al.; Licensee Bentham Open.

This is an open access article licensed under the terms of the Creative Commons Attribution Non-Commercial License (http://creativecommons.org/licenses/by-nc/3.0/) which permits unrestricted, non-commercial use, distribution and reproduction in any medium, provided the work is properly cited. 